\title{
The effectiveness of chemotherapy for treatment of high grade astrocytoma in children: Results of a randomized trial A report from the Childrens Cancer Study Group
}

\author{
Richard Sposto ${ }^{1}$, Inta J. Ertel ${ }^{2}$, R.D.T. Jenkin ${ }^{3}$, Carl P. Boesel ${ }^{4}$, Joan L. Venes ${ }^{5}$, Jorge A. Ortega ${ }^{6}$, \\ Audrey E. Evans ${ }^{7}$, William Wara ${ }^{8}$ and Denman Hammond ${ }^{1}$ \\ ${ }^{1}$ University of Southern California School of Medicine, Los Angeles; ${ }^{2}$ University of Michigan, Ann Arbor; \\ ${ }^{3}$ Toronto-Bayview Cancer Center; ${ }^{4}$ Ohio State University, Columbus; ${ }^{5}$ Mott Childrens Hospital, Ann Arbor; \\ ${ }^{6}$ Childrens Hospital of Los Angeles; ${ }^{7}$ Children's Hospital of Philadelphia; ${ }^{8}$ University of California, \\ San Francisco, School of Medicine
}

Key words: astrocytoma, glioblastoma multiforme, children, chemotherapy, randomized trial

\section{Summary}

Fifty-eight patients with high-grade astrocytoma were treated by members of the Childrens Cancer Study Group in a prospective randomized trial designed to study the effectiveness of chemotherapy as an adjuvant to standard surgical treatment and radiotherapy. Following surgical therapy, patients were assigned randomly to radiotherapy with or without chemotherapy consisting of chloroethyl-cyclohexyl nitrosourea, vincristine, and prednisone. Treatment with chemotherapy prolonged survival and event-free survival. Five-year event-free survival was $46 \%$ for patients in the radiotherapy and chemotherapy group, and $18 \%$ for patients in the radiotherapy-alone group. Five-year survival was similarly improved. The differences in outcome due to treatment were statistically significant after correcting for imbalances in important prognostic factors (event-free survival, $\mathrm{p}=0.026$; survival, $\mathrm{p}=0.067$ ). The presence of mitoses or necrosis in the tumor specimen was associated with poorer outcome. Patients whose initial surgery was limited to biopsy, and patients with basal ganglia lesions, also had significantly worse outcome. Chemotherapy administered at the time of recurrence in a small number of patients did not produce any long-term survivors. This study is to our knowledge the only randomized trial to investigate effectiveness of chemotherapy in the treatment of high-grade astrocytoma in children.

\section{Introduction}

Astrocytomas represent approximately $37 \%$ of all childhood brain tumors and $8 \%$ of all cancers in young people. As a group these tumors are morphologically and biologically diverse, and consequently display various degrees of malignancy. It is however customary to distinguish between two classes of astrocytomas. Low-grade astrocytomas (astrocytoma grade I-II), which comprise roughly $75 \%$ of astrocytomas in children, are usually welldifferentiated, slow growing tumors which arise most frequently in the cerebellum. These are often treated successfully with surgery alone. In contrast,

Contributing Childrens Cancer Study Group investigators, institutions, and grant numbers are given in the appendix. Grant support from the Division of Cancer Treatment, National Cancer Institute, National Institutes of Health, Department of Health and Human Resources. 
high-grade astrocytomas (astrocytoma grade IIIIV, glioblastoma multiforme), which are the most common brain tumors in adults, are rapidly growing, relatively poorly differentiated tumors, fiftyfive percent of which occur supratentorially, $5 \%$ in the cerebellum, and $40 \%$ in the brain stem. Partial surgical excision of supratentorial and cerebellar high-grade astrocytoma is often possible, but this alone rarely produces adequate disease control. Hence, post-surgical radiation therapy is routinely employed. The prognosis for high-grade astrocytoma treated with surgery and radiotherapy alone is nevertheless poor. In general, the mean duration of survival following diagnosis is less than a year [1-5].

In 1976, in an attempt to improve the survival of children with high-grade astrocytomas, the Childrens Cancer Study Group initiated a randomized clinical trial, CCG-943, to study the effectiveness of vincristine (VCR), chlorethyl-cyclohexyl nitrosourea (CCNU) and prednisone (PDN) in prolonging survival and event-free survival when added to standard radiation therapy. A second goal of the trial was to determine the effectiveness of procarbazine (PCZ) to induce response in recurrent patients. CCNU, VCR, and PCZ were chosen because each has a different mechanism of action. CCNU is a small, lipid soluble drug which is able to cross the blood-brain barrier. Its antitumor effect is derived primarily through alkylation. Procarbazine is a monoamine oxidase inhibitor which is thought to behave as an alkylating agent. It also shows the ability to cross the blood-brain barrier. VCR acts by blocking mitosis with metaphase arrest, but has only limited ability to cross the blood-brain barrier. It may nevertheless be effective against portions of the tumor in which the blood-brain barrier is disrupted. All have shown the ability to induce tumor responses in one or more intracranial neoplasms [6-9]. Prednisone was included to minimize undesirable neurological reactions to initiation or withdrawal of drug therapy due to cerebral edema, and for the possibility that its effect on cell membrane permeability might enhance the action of the other chemotherapeutic agents.

\section{Methods}

Eligibility, staging, and randomization

All newly diagnosed patients between the ages of 2 and 21 years with biopsy confirmed intracranial high-grade astrocytoma (Kerhonan [10] grades IIIII, III, III-IV, and IV) were eligible. Patients with primary brain stem or spinal cord lesions were not eligible for this study. The study was divided into two phases. In Phase I, following initial surgery and pathologic confirmation of diagnosis, eligible patients were assigned randomly to receive either radiotherapy alone or radiotherapy with one year of chemotherapy consisting of CCNU, VCR, and PDN. Patients who showed evidence of disease progression or recurrence were eligible to enter Phase II and receive therapy consisting of PCZ for patients who had received CCNU, VCR, and PDN in Phase I, or CCNU, VCR, and PCZ for those who had received radiotherapy alone. Phase II of the study was included to standardize the treatment after first recurrence or progression of disease and to assess the ability of PCZ to induce a response in patients with recurrent disease.

Disease status was determined on the basis of history, physical exam, brain scan, and operative findings. The use of other studies (e.g., EMI, CT, CSF cytology, myelogram) was at the discretion of the treating physician as needed for diagnosis. CT scans were not universally available at the beginning of the study and hence were not required, although staging and follow-up of many patients did include CT.

Guidelines for surgical treatment encouraged as complete a resection as possible to provide adequate internal decompression and to reduce total tumor burden when this was compatible with good post-operative neurological function. Dexamethasone was used in the pre- and post-operative period according to standard neurosurgical practice, but was to be tapered and discontinued as soon as possible, preferably within the first two weeks of therapy. Surgical checklists and surgical notes were reviewed centrally and patients were classified as having complete resection, partial resection, or biopsy only. 
Central pathology review was performed on all patients. If the original diagnosis of astrocytoma grade II-III or greater was not supported by the study reviewer, the patient was declared ineligible. When pathology material was not available or not evaluable, the patient was excluded from all analyses. In the final review, tumors were classified as either anaplastic astrocytoma (AA) or glioblastoma multiforme (GBM), the primary distinction between these being the presence in the latter of at least one focus of unequivocal necrosis involving neoplastic astrocytes [11]. Mitotic figures were considered present if more than one mitosis was observed per ten high-power fields [12].

Randomization and the start of treatment were required within 4 weeks of surgery. An adaptive randomization procedure [13] was used to balance the two treatment groups with respect to extent of resection (total, partial, and biopsy) and site of disease (supratentorial or infratentorial).

\section{Radiation therapy}

Both treatment regimens included radiotherapy to a dose of 5250 rad in 28 fractions delivered 5 days week. This dose was reduced to $4500 \mathrm{rad}$ in 28 fractions for children 2 to 3 years of age. Radiotherapy guidelines specified that the whole tumor volume, as defined by neuroradiological investigation and surgical exploration, plus a $4 \mathrm{~cm}$ margin of 'normal' brain parenchyma, would be irradiated. In cerebellar lesions, the lower surface of the body of $\mathrm{C} 2$ would be the field limit. A minimum field size of $100 \mathrm{~cm}^{2}$ was required. For large tumors investigators often interpreted these criteria as a requirement for whole brain irradiation. The tumor dose was defined as the mid-plane dose on the central axis for equally weighted parallel opposed beams, and as the most frequent dose in the tumor volume (i.e. the modal dose) for unequally weighted parallel opposed beams and for other methods of irradiation. Dose variation within the tumor volume was limited to within $5 \%$ of the prescribed tumor dose. Incremental escalation to the prescibed daily radiation dose was permitted during the first 4 days of treatment. The radiation beam energy was defined as cobalt 60 or higher. Institutional radiation oncologists submitted radiation therapy checklists, radiation treatment sheets and the isodose distributions for central review.

\section{Phase I chemotherapy}

The experimental treatment regimen consisted of radiotherapy as described above with the addition of chemotherapy during and following radiation for a total treatment duration of 58 weeks. Vincristine was started within four weeks of diagnosis at $1.5 \mathrm{mg} / \mathrm{m}^{2}$ intravenously (I.V.) per week (maximum $2 \mathrm{mg}$ ) for six injections throughout radiotherapy. After an interval of four weeks following the completion of radiation therapy, six-week chemotherapy cycles were initiated. Vincristine was given on days 1,8 and 15 of each cycle. CCNU $100 \mathrm{mg} / \mathrm{m}^{2}$ was given by mouth (p.o.), before breakfast, on day 2 of each cycle. Prednisone $40 \mathrm{mg} / \mathrm{m}^{2} /$ day in three divided doses was given p.o. for the first 14 days of each cycle. Dexamethasone was not administered concurrently with prednisone.

\section{Treatment of recurrent or progressive disease}

Disease progression or recurrence were defined as abnormal symptoms accompanied by new or deteriorating neurological signs, or documented changes in neuroradiological examinations such as CT scan or myelogram. Investigators were urged to wait as long as possible until recurrent or progressive disease was indisputable before declaring a recurrence.

Patients who entered Phase II of the study after documentation of recurrent or progressive disease were retreated with radiation and chemotherapy as follows. Patients who were treated initially with chemotherapy and radiation received $\mathrm{PCZ}$ at $100 \mathrm{mg} / \mathrm{m}^{2} /$ day p.o. for 14 days of each six-week cycle for eight cycles (starting at $50 \mathrm{mg} / \mathrm{m}^{2}$ in the first cycle and increasing by $20 \%$ in subsequent cycles to full dose in the absence of toxicity). $\mathrm{Pa}-$ tients who were treated initially with radiation alone received VCR and CCNU at the same dose 
and schedule as in Phase I. PDN was replaced with PCZ at $100 \mathrm{mg} / \mathrm{m}^{2} /$ day p.o. for 14 days of each six week cycle (starting at $50 \mathrm{mg} / \mathrm{m}^{2} / \mathrm{day}$ ). Therapy would continue for eight cycles. Patients who recurred after 12 months could optionally receive radiation therapy to recurrence sites to a dose of $3000 \mathrm{rad}$ in $15-18$ fractions.

\section{Statistical methods}

Analysis of patient and disease characteristics and treatment effect were based on event-free survival (EFS), the time from study entry to the first disease progression, disease recurrence, or death, and survival, the time from study entry to death. EFS was censored at the time of last patient contact at which disease status could be ascertained. Survival was censored at the time of last patient contact. Survival and EFS were not censored, nor were patients excluded from analysis, because of deviations from protocol therapy. Statistical hypothesis tests for survival and EFS were based on the logrank statistic. The P-values for treatment effect were computed using both unstratified and stratified versions of the logrank test, the latter to reduce bias in the treatment comparison attributable to imbalances in important prognostic factors $[14,15]$. P-values quoted in the text and figures are for two-sided tests. Product-limit (Kaplan-Meier) estimates of survival and event-free survival functions are used in the figures and to obtain survival and EFS probabilities quoted in the text. Standard errors for the latter are derived from Greenwood's formula [16]. Follow-up data to July 1, 1986 were included in the analysis. This date is five years after the last patient entry. All analyses of treatment effects were performed both according to randomized assignment irrespective of treatment received, and including only patients who received the assigned treatment $[17,18]$.

Three patients who died lacked data on the time of recurrence and were censored for EFS. All EFS analyses were repeated with these patients considered as events at the censoring time, and as events at the time of death. Differences between these analyses are discussed where appropriate.

\section{Results}

Between 1976 and 1981, 72 patients were enrolled on the CCG-943 high-grade astrocytoma study. Thirteen patients were subsequently declared ineligible. Three of these were previously treated or relapsed patients who were entered only on Phase II of the study; three were found not to be astrocytomas (two ependymomas, one microglial sarcoma); two had insufficient material for histologic review; three were low-grade astrocytomas; for one the tumor was located in the spine at the level of C5-C6; and one withdrew from the study before treatment was started.

Fifty-eight of 59 patients were randomized, 28 to receive radiotherapy and chemotherapy (RT + $\mathrm{CRX}$ ), and 30 to receive radiotherapy alone (RT). Three patients were treated with chemotherapy after initial assignment to RT. One of these received treatment with MOPP therapy after completing radiation. These patients were retained in most analyses $[17,18]$ as ones who were randomized to RT but switched to RT + CRX, but in one analysis were excluded. One of the patients in the chemotherapy group received Phase II chemotherapy with no additional radiotherapy after an apparent recurrence at 9 months, which in retrospect was the result of a CT artifact. This patient was alive and disease free at last follow-up with CT similar to that before and at the time of the declared recurrence, and is retained uncensored in the analysis. The non-randomized patient was treated with RT + CRX. This report will deal only with the 58 randomized patients from this study.

Characteristics of the 58 patients are summarized in Table 1 . The two randomized groups are well balanced with respect to all factors, except that a higher proportion of patients treated with RT alone are glioblastoma multiforme $(p=0.030)$. Mitosis and necrosis are correlated with the pathologic classification and are therefore similarly unbalanced. The statistical comparison of treatment groups was corrected for these imbalances. 


\section{Analysis of patient and disease characteristics}

The results of survival and EFS analyzed by individual patient and disease characteristics are shown in Table 2. Age, sex, and race were not significantly associated with either survival or EFS. However, pathologic classification, primary site of disease, extent of resection, necrosis, and mitosis were significantly associated with both types of outcome.

Extent of resection and site of disease were high- ly correlated. Surgery was limited to biopsy only in $9.5 \%(4 / 42)$ of patients with hemispheric lesions and $0.0 \%(0 / 7)$ with posterior fossa lesions, as compared with $77.8 \%(7 / 9)$ of patients with lesions in the basal ganglia $(\mathrm{p}<0.001$, chi-square test for homogeneity. Diencephalic lesions are also included in the 'basal ganglia' category.) Both site of disease and extent of resection were significantly correlated with survival and EFS $(p<0.001)$. Survival by site of disease is shown in Fig. 1. Patients with

Table 1. Distribution of patient and disease characteristics within randomized groups. Frequency (percent)

\begin{tabular}{|c|c|c|c|c|c|c|}
\hline & \multicolumn{6}{|c|}{ Randomized regimen } \\
\hline & \multicolumn{2}{|c|}{$\mathrm{RT}+\mathrm{CRX}$} & \multicolumn{2}{|c|}{ RT alone } & \multicolumn{2}{|l|}{ Total } \\
\hline & $\mathrm{N}=28$ & $(\%)$ & $\mathrm{N}=30$ & $(\%)$ & $N=58$ & $(\%)$ \\
\hline \multicolumn{7}{|l|}{ Treated with } \\
\hline $\mathrm{RT}+\mathrm{CRX}$ & 28 & $(100)$ & 3 & $(10)$ & 31 & (53) \\
\hline RT alone & 0 & (0) & 27 & $(90)$ & 27 & $(47)$ \\
\hline \multicolumn{7}{|l|}{ Age } \\
\hline Under 4 years & 2 & (7) & 3 & (10) & 5 & ( 8 ) \\
\hline 4 to 6 years & 6 & (22) & 8 & (27) & 14 & (24) \\
\hline 7 to 10 years & 4 & (14) & 6 & $(20)$ & 10 & (17) \\
\hline Over 10 years & 16 & (59) & 13 & (43) & 29 & $(50)$ \\
\hline \multicolumn{7}{|l|}{ Sex } \\
\hline Male & 15 & (54) & 17 & $(57)$ & 32 & (55) \\
\hline Female & 13 & (46) & 13 & (43) & 26 & $(45)$ \\
\hline \multicolumn{7}{|l|}{ Race } \\
\hline White & 21 & (75) & 26 & (87) & 47 & $(81)$ \\
\hline Black & 4 & (14) & 3 & (10) & 7 & $(12)$ \\
\hline Other & 3 & (11) & 1 & (3) & 4 & (7) \\
\hline \multicolumn{7}{|l|}{ Extent of resection } \\
\hline Complete & 3 & (11) & 5 & (17) & 8 & (14) \\
\hline Partial & 20 & (71) & 19 & $(63)$ & 39 & $(67)$ \\
\hline Biopsy & 5 & (18) & 6 & (20) & 11 & (19) \\
\hline \multicolumn{7}{|l|}{ Pathology classification } \\
\hline Anaplastic & 13 & (46) & 5 & (17) & 18 & (31) \\
\hline Glioblastoma multiforme & 15 & (54) & 25 & (83) & 40 & (69) \\
\hline \multicolumn{7}{|l|}{ Primary site of disease } \\
\hline Hemisphere & 20 & (72) & 22 & (73) & 42 & (72) \\
\hline Posterior Fossa & 4 & (14) & 3 & $(10)$ & 7 & (12) \\
\hline Basal Ganglia & 4 & (14) & 5 & (17) & 9 & (16) \\
\hline \multicolumn{7}{|l|}{ Mitosis } \\
\hline Positive & 12 & (43) & 22 & (73) & 34 & $(59)$ \\
\hline Negative & 13 & (46) & 7 & (24) & 20 & (34) \\
\hline Not evaluable & 3 & (11) & 1 & (3) & 4 & (7) \\
\hline \multicolumn{7}{|l|}{ Necrosis } \\
\hline Positive & 16 & (57) & 24 & $(80)$ & 40 & (69) \\
\hline Negative & 10 & (36) & 5 & (17) & 15 & (26) \\
\hline Not evaluable & 2 & (7) & 1 & (3) & 3 & $(5)$ \\
\hline
\end{tabular}




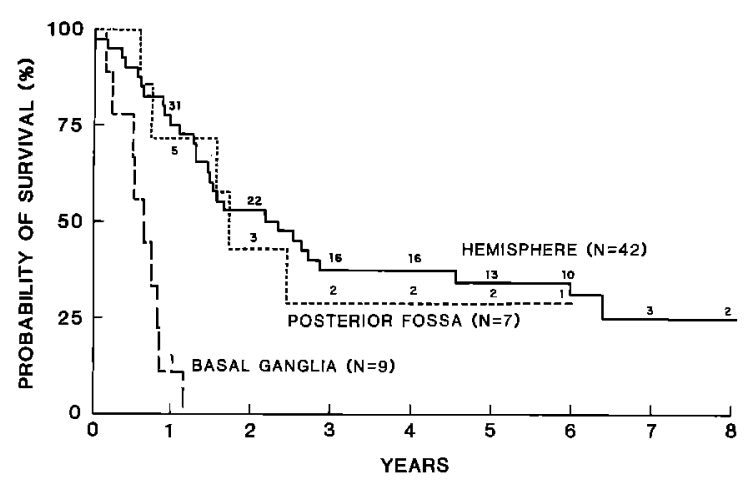

Fig. 1. Survival (in years) by site of disease.

primary site in the basal ganglia had an extremely poor outcome. No difference in outcome between hemispheric and posterior fossa sites of disease was observed. Survival by extent of resection is shown in Fig. 2, which demonstrates that patients with surgery limited to biopsy had a very poor outcome. Patients with basal ganglia tumors were in many cases the same patients as those with surgery limited to biopsy. Consequently, one cannot determine clearly which of these factors is a more important predictor of outcome.

Pathologic classification, necrosis, and mitosis were also highly correlated. Although the primary distinction between AA and GBM is the presence of necrosis in the latter, in two cases other features of the tumor were thought to suggest a classification opposite to that indicated by the presence or

Table 2. Analysis of survival and event-free survival by patient and disease characteristics ${ }^{\mathrm{a}} \mathrm{N}=58$

\begin{tabular}{lrr}
\hline & $\begin{array}{l}\text { Survival } \\
\text { P-value }^{\text {b }}\end{array}$ & $\begin{array}{l}\text { Event-free survival } \\
\text { P-value }\end{array}$ \\
\hline Age & 0.389 & 0.344 \\
Pathology & 0.012 & 0.019 \\
Primary site of disease & $<0.001$ & $<0.001$ \\
Extent of resection & $<0.001$ & $<0.001$ \\
Sex & 0.109 & 0.122 \\
Race & 0.430 & 0.628 \\
Necrosis & 0.034 & 0.049 \\
Mitosis $^{\mathrm{c}}$ & 0.058 & 0.078 \\
\hline
\end{tabular}

See Table 1 for categories.

b Two-sided P-values for unstratified logrank test.

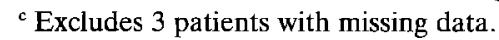

${ }^{d}$ Excludes 4 patients with missing data.

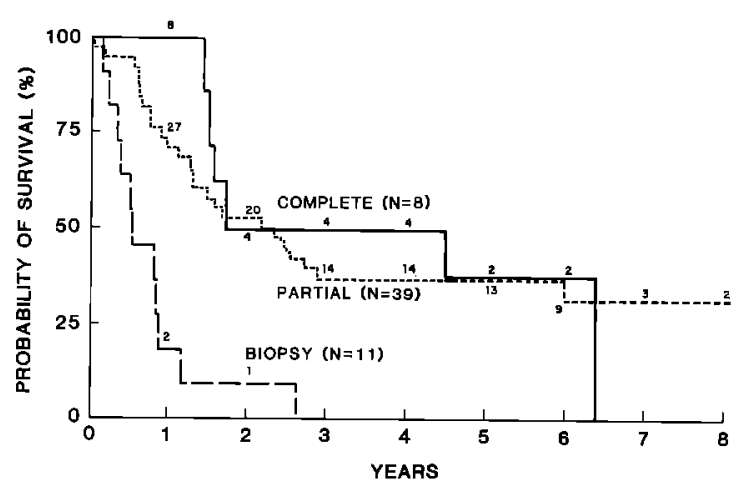

Fig. 2. Survival (in years) by extent of resection.

absence of necrosis. Only $13.3 \%(2 / 15)$ of AA tumors displayed mitoses as compared with $80 \%$ (32/ 40) of GBM tumors. In 55 tumors with data on mitosis and necrosis, $43(78.2 \%)$ agreed on the presence or absence of these features $(p<0.001$, chi-square test for homogeneity).

Survival by pathologic classification is shown in Fig. 3. Patients with glioblastoma multiforme have significantly shorter survival than those with anaplastic astrocytoma $(p=0.012)$. Similar results are obtained when the sample is divided into groups based on either mitosis or necrosis. Here also one cannot choose one of these variables as the most suitable predictor of prognosis.

\section{Analysis of treatment effect on outcome}

Eighteen patients in the chemotherapy group and 25 in the radiation-only group died of progressive disease. Three of these were censored for EFS, two in the chemotherapy group and one in the radiotherapy group. All other patients were alive and presumably disease-free at last follow-up. Figs 4 and 5 show survival and EFS by randomized treatment assignment. Five-year survival was $43 \%(+/$ $-9 \%$ ) for patients randomized to radiotherapy and chemotherapy, and $17 \%(+/-7 \%)$ for patients randomized to radiotherapy alone. Five-year event-free survival was $46 \%(+/-10 \%)$ and $18 \%$ $(+/-7 \%)$ respectively. Event-free survival was greater numerically than survival because of the three censored patients mentioned above. Fiveyear EFS and survival figures changed only margin- 


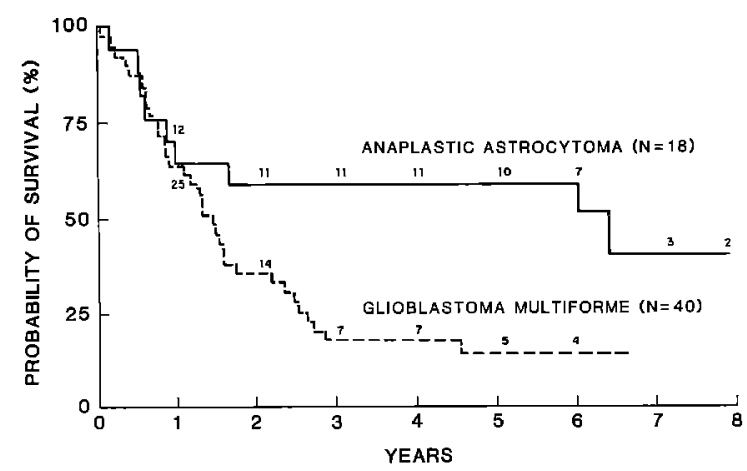

Fig. 3. Survival (in years) by review pathology classification.

ally if patients who switched therapy after randomization (including the MOPP-treated patient) were excluded, or if the three patients who were censored for EFS were assumed to have recurred at the time of censoring, or at the time of death.

The majority of treatment failures involved local tumor progression or recurrence, some concurrent with new regional or metastatic lesions. In only four cases did recurrent disease manifest itself by lesions in previously uninvolved sites alone: one isolated bone, one spinal, one lateral ventricular metastasis in a patient with primary disease localized to a cerebellar hemisphere, and one late (after five years) recurrence in the brain stem in a patient with a primary temporal lesion.

Table 3A gives P-values for the comparison of randomized treatment groups. The unstratified comparison based on EFS was significant with $\mathrm{P}<0.05$ whether or not patients who switched treatment were included. The comparison of survival is significant at the $\mathrm{P}<0.10$ level. Table $3 \mathrm{~B}$ shows the results when the data were stratified by the important prognostic factors pathology and extent of resection. EFS remained significant at $\mathrm{P}<0.05$ and survival at $\mathrm{P}<0.10$. When the patients who were censored for EFS were analyzed in the various ways discussed in 'Statistical Consideration', all EFS comparisons remained significant at $\mathrm{P}<0.05$.

Fig. 6 shows event-free survival by randomized assignment separately within pathology groups for patients who received at least a partial resection. The difference between treatment groups is pronounced in GBM patients. In this group, five-year

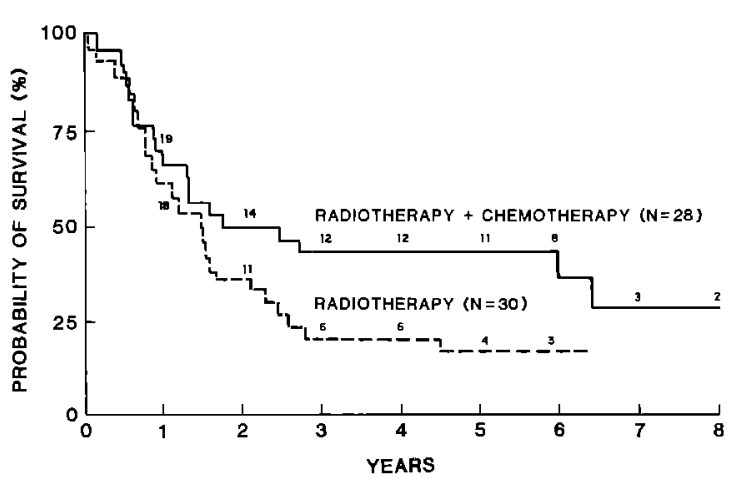

Fig. 4. Survival (in years) by randomized treatment assignment.

event-free survival was $42 \%$ in patients treated with RT + CRX and $6 \%$ when treated with RT $(p=0.011)$. Five-year survival in this group was $36 \%$ for RT + CRX and $5 \%$ for RT $(p=0.044)$. A benefit from chemotherapy is not observed in AA patients. Patients with biopsy only all failed rapidly regardless of treatment or histology, and are not shown.

\section{Radiotherapy review}

Radiotherapy was reviewed for 51 patients. Adequate radiotherapy data on the remaining eight patients could not be obtained. Eighty-two percent $(42 / 51)$ of the reviewed patients received radiation doses within $10 \%$ of the nominal protocol dose. Nine patients received doses which deviated by more than $10 \%$. Two patients had very large deviations - one patient was assigned to receive radiotherapy and chemotherapy but refused treatment partway through radiotherapy and died at 6 months, the other died during radiotherapy. The radiotherapy dose distributions in the two treatment groups were comparable. A smaller proportion of patients treated with $\mathrm{RT}+\mathrm{CRX}$ received whole brain irradiation as compared with patients treated with $\mathrm{RT}(11 / 28$ vs. $15 / 23, \mathrm{p}=0.10)$. The majority of patients were treated with either 4 MEV or Cobalt 60 equipment. None of these factors had an apparent effect on survival or eventfree survival analyses. 


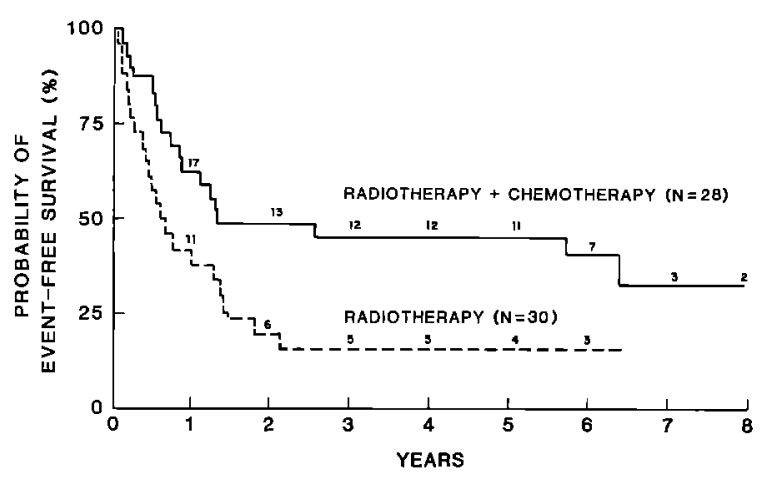

Fig. 5. Event-free survival (in years) by randomized treatment assignment.

\section{Phase I chemotherapy review}

Most of the 31 patients who were treated with Phase I chemotherapy had completed a proportion of chemotherapy consistent with their time on Phase I. Therapy modifications or delays were reported in 19 patients. Therapy delays or omission of one or more drug doses were noted in 10 patients because of infection, in three because of decreased absolute neutrophil count (ANC) or platelets, and in two for unknown reasons. Five infections were observed without low blood counts: 1 osteomyelitis, 2 pneumonia, 1 aseptic meningitis, 1 chicken pox. Vincristine doses during radiotherapy were interrupted for 5 weeks in one patient because of progressive visual loss, and were omitted in the last three cycles in one patient who experienced psychomotor seizures. Prednisone was omitted in one patient in cycles 4 through 8 during administration of dexamethasone, and was discontinued after cycle 1 in a patient who could not swallow the tablets.

Table 3. Analysis of treatment effect on survival and event-free survival (Entries are two-sided P-values for the logrank test)

\begin{tabular}{|c|c|c|c|c|}
\hline & \multicolumn{2}{|c|}{ A. Unstratified } & \multicolumn{2}{|c|}{ B. Stratified ${ }^{\mathrm{a}}$} \\
\hline & Survival & EFS & Survival & EFS \\
\hline $\begin{array}{l}\text { - As randomized } \\
\quad(\mathrm{N}=58)\end{array}$ & 0.080 & 0.021 & 0.067 & 0.026 \\
\hline $\begin{array}{l}\text { - Treated as randomized } \\
\text { only }(\mathrm{N}=55)\end{array}$ & 0.086 & 0.021 & 0.067 & 0.025 \\
\hline
\end{tabular}

${ }^{\mathrm{a}}$ Logrank test stratified by Pathology and Extent of Resection.

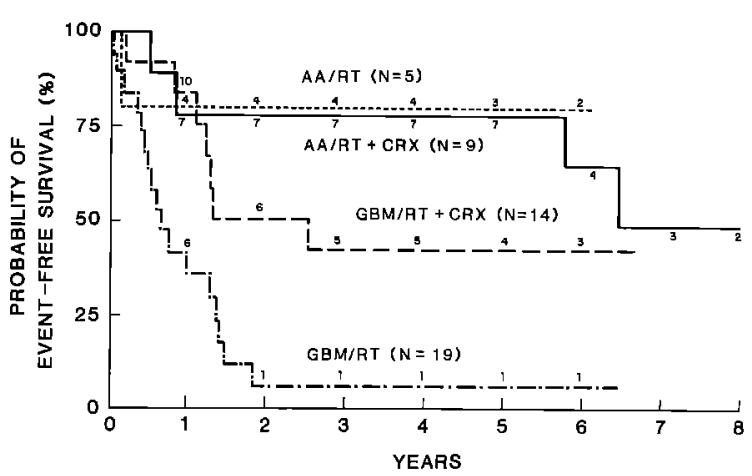

Fig. 6. Event-free survival (in years) by pathology and randomized treatment assignment. Patients with complete + partial resection only. AA, Analplastic Astrocytoma; GBM, Glioblastoma Multiforme; RT, Radiation therapy alone; RT + CRX, Radiation therapy plus chemotherapy.

One patient mentioned in the previous section refused all therapy after one month.

Thirteen patients completed Phase I chemotherapy in an average of 57 weeks. One patient received an extra cycle. Eight of the 13 were alive without signs of recurrence or progression an average of 6.6 years from diagnosis. Four of the remaining five showed signs of recurrence 2, 4, 17 and 59 months after completing chemotherapy and subsequently expired, the last being a brain stem recurrence in a temporal anaplastic astrocytoma on which no post-mortem was performed. The fifth patient died of shunt complications at 64 months but was apparently free of tumor. Eighteen patients did not complete Phase I chemotherapy; 10 exhibited progression or recurrent disease on therapy and subsequently expired, four refused further protocol therapy at 2 weeks, 3 weeks, 3 months, and 12 months and expired at 6, 5, 8, and 32 months. Treatment was halted after 5 cycles in one patient because of abdominal pain and abnormal liver function tests and never resumed. This patient is alive and disease-free at 6.6 years. One patient died of progressive disease at 2 months after treatment was terminated at 1 month because of pneumonia. The patient who was incorrectly declared a recurrence was given Phase II chemotherapy and is alive at 6.4 years. The patient who received 12 cycles of MOPP therapy is alive and disease-free at 5.6 years. 


\section{Phase II chemotherapy}

Forty-three patients died of tumor recurrence, progressive disease, or complications due to disease. The treatment after disease progression is not known in eight of $43 ; 20$ received no subsequent treatment either because of parental refusal, or because of rapid deterioration which precluded further therapy; four were treated on CCSG new agents protocols (three with cisplatinum, one with AZQ); two received other non-protocol therapy; and nine were treated with Phase II therapy. Five who were treated initially with RT received $1,3,5$, 8 , and 9 cycles of chemotherapy consisting of vincristine, CCNU, and procarbazine. Two exhibited some indication of improvement, but all subsequently died of progressive disease. Mean survival after recurrence in these patients was 14.6 months. Four patients who were treated initially with RT + CRX received 1, 2, 2, and 3 cycles of Phase II therapy consisting of procarbazine alone. None of these patients exhibited detectable benefit from Phase II therapy, and all died of progressive disease with a mean survival of 7.7 months after recurrence. The difference in median post-recurrence survival is marginally significant ( $p=0.11$, permutation test). Mean post-recurrence survival in five patients originally treated with RT who received other post-recurrence treatment was 7.8 months, which is less than for RT patients treated with Phase II therapy $(\mathrm{p}=0.087)$.

\section{Discussion}

This study demonstrates that chemotherapy consisting of CCNU, vincristine, and prednisone, when added to standard surgery and radiotherapy, may prolong event-free survival and survival of children with supratentorial or cerebellar highgrade astrocytoma. The difference in EFS and survival between treatment groups was largest in patients with glioblastoma multiforme with at least partial resection of tumor. A difference between treatment groups was not observed in patients with anaplastic astrocytoma, or in patients who underwent biopsy only.
The ability to achieve at least a partial tumor resection was associated with better outcome. Patients whose surgery was limited to biopsy had significantly worse outcome. We found no difference, either in event-free survival or survival, when patients who had undergone grossly complete resections were compared with those with partial resections. The primary contributing factor to the poor outcome of the biopsy-only patients may have been either the amount of tumor remaining after surgery, or the initial tumor size or location. We could not determine from our data which of these two factors was more important. Very large or deeply seated tumors can interfere with critical brain structures and therefore contribute to poor outcome. These tumors can also, by virtue of their size or location, limit the surgeon's ability to reduce tumor bulk.

Histological features of the tumor, in particular the distinction between anaplastic astrocytoma and glioblastoma multiforme, were important determinants of prognosis in these patients. The presence of either necrosis or mitosis in the tumor specimen was associated significantly with poorer outcome. This finding is consistent with reports on the importance of necrosis or mitosis as prognostic criteria in adult malignant glioma [11, 12, 19] and in childhood brain stem glioma [20]. This class of neoplasms displays heterogeneity in other histologic characteristics [10] which were not studied. Mitosis and necrosis represent two of many features which may be associated with degree of malignancy in these tumors, or to their susceptibility to chemotherapy or other forms of treatment.

It is difficult to draw reliable conclusions about the efficacy in recurrent astrocytoma of procarbazine alone or in combination with CCNU and vincristine based on the relatively few patients in the non-randomized, Phase II portion of this study. Treatment with CCNU, vincristine, and procarbazine in patients initially treated with RT produced longer survival than other post-recurrence chemotherapy. This is consistent with the belief that these agents are active for treatment of high-grade astrocytoma. However, no long-term survivals resulted. Delay in chemotherapy until the time of recurrence is therefore not supported. 
One should take care when interpreting the treatment comparison which we reported for individual patient subgroups. Chemotherapy significantly prolonged event-free survival and survival in patients with glioblastoma multiforme who received at least a partial resection of their tumor, but had no apparent effect in patients with anaplastic astrocytoma or biopsy only. This seems to imply that chemotherapy is only of benefit in the first group. There were, however, relatively few patients in the biopsy-only and anaplastic astrocytoma patient subgroups. Furthermore, the discrimination between biopsy-only and partially resected patients, or between anaplastic astrocytoma and glioblastoma multiforme, represents cutpoints on a continuum rather than unequivocal distinctions. Because of this it could equally be argued that chemotherapy may be effective to some extent in biopsy-only and anaplastic astrocytoma categories even though we did not observe an outcome difference due to treatment. This question cannot be resolved from our data alone.

CCG-943 is to our knowledge the only randomized clinical trial to investigate the effectiveness of chemotherapy in the treatment of high grade astrocytoma in children. A number of trials have investigated the efficacy of chemotherapy in treating adult malignant glioma. Nine clinical trials reported between 1976 and 1985 [21-29] included randomized comparisons of radiotherapy with and without chemotherapy containing a nitrosourea alone or in combination with other agents. Two of these $[21,25]$ reported significant prolongation of survival, although in one [21] the importance of CCNU in the chemotherapy regimen could not be demonstrated clearly. In one report [22], survival was prolonged significantly in a subset of patients. In three reports $[22,28,29]$, a significantly greater number of long-term survivors were reported in the chemotherapy-treated group. In the remaining studies, no advantage to chemotherapy when added to radiotherapy was found. None of the trials reported significantly poorer survival in the chemotherapy group. Stenning et al. [30] concluded from their thorough review that reports in the medical literature provide evidence that a course of nitrosourea only, when added to radiotherapy, improves survival of high grade glioma patients. Hence, although the collective findings of the reported trials and ours are mixed, they nevertheless support the view that chemotherapy can improve survival and event-free survival in the treatment of intracranial high-grade astrocytoma.

\section{Acknowledgments}

The authors would like to thank Dr. Jonathan Finlay, Dr. Nathan Kobrinsky, and numerous other CCSG investigators for their detailed reviews of earlier versions of this paper. 


\section{Appendix. Principal Investigators Childrens Cancer Study Group}

\begin{tabular}{|c|c|c|}
\hline Institution & Investigator & Grant No. \\
\hline $\begin{array}{l}\text { Group Operations Office } \\
\text { University of Southern California } \\
\text { Comprehensive Cancer Center } \\
\text { Los Angeles, California }\end{array}$ & $\begin{array}{l}\text { Denman Hammond, M.D. } \\
\text { John Weiner, Dr P.H. } \\
\text { Harland Sather, Ph.D. } \\
\text { Richard Sposto, Ph.D. } \\
\text { Mark Krailo, Ph.D. } \\
\text { Jonathan Buckley, M.B.B.S., Ph.D. } \\
\text { Madeline Bauer, Ph.D. }\end{array}$ & CA 13539 \\
\hline $\begin{array}{l}\text { University of California Medical Center } \\
\text { San Francisco, California }\end{array}$ & Arthur Ablin, M.D. & CA 17829 \\
\hline $\begin{array}{l}\text { University of Wisconsin Hospital } \\
\text { Madison, Wisconsin }\end{array}$ & Paul Gaynon, M.D. & CA 05436 \\
\hline $\begin{array}{l}\text { Children's Orthopedic Hospital and Medical Center } \\
\text { Seattle, Washington }\end{array}$ & Ronald Chard, M.D. & CA 10382 \\
\hline $\begin{array}{l}\text { Rainbow Babies and Children's Hospital } \\
\text { Cleveland, Ohio }\end{array}$ & Susan Shurin, M.D. & CA 20320 \\
\hline $\begin{array}{l}\text { Children's Hospital National Medical Center } \\
\text { Washington, D.C. }\end{array}$ & Gregory Reaman, M.D. & CA 03888 \\
\hline $\begin{array}{l}\text { Children's Hospital of Los Angeles } \\
\text { Los Angeles, California }\end{array}$ & Jorge Ortega, M.D. & CA 02649 \\
\hline $\begin{array}{l}\text { Children's Hospital of Columbus } \\
\text { Columbus, Ohio }\end{array}$ & Frederick Ruymann, M.D. & CA 03750 \\
\hline $\begin{array}{l}\text { Children's Hospital of Pittsburgh } \\
\text { Pittsburgh, Pennsylvania }\end{array}$ & Vincent Albo, M.D. & CA 36015 \\
\hline $\begin{array}{l}\text { Vanderbilt University School of Medicine } \\
\text { Nashville, Tennessee }\end{array}$ & John Lukens, M.D. & CA 26270 \\
\hline $\begin{array}{l}\text { University of Minnesota Health Sciences Center } \\
\text { Minneapolis, Minnesota }\end{array}$ & William Woods, M.D. & CA 07306 \\
\hline $\begin{array}{l}\text { Memorial Sloan-Kettering Cancer Center } \\
\text { New York, New York }\end{array}$ & Peter Steinherz, M.D. & CA 42764 \\
\hline $\begin{array}{l}\text { James Whitcomb Riley Hospital for Children } \\
\text { Indianapolis, Indiana }\end{array}$ & Robert Weetman, M.D. & CA 13809 \\
\hline $\begin{array}{l}\text { Hospital for Sick Children } \\
\text { Toronto, Ontario, Canada }\end{array}$ & Alvin Zipursky, M.D. & - \\
\hline $\begin{array}{l}\text { University of British Columbia } \\
\text { Vancouver, British Columbia, Canada }\end{array}$ & Paul Rogers, M.D. & CA 29013 \\
\hline $\begin{array}{l}\text { Children's Hospital Medical Center } \\
\text { Cincinnati, Ohio }\end{array}$ & Beatrice Lampkin, M.D. & CA 26126 \\
\hline $\begin{array}{l}\text { Harbor/UCLA \& Miller Children's Medical Center } \\
\text { Torrance and Long Beach, California }\end{array}$ & Jerry Finklestein, M.D. & CA 14560 \\
\hline $\begin{array}{l}\text { University of California Medical Center } \\
\text { Los Angeles, California }\end{array}$ & Stephen Feig, M.D. & CA 27678 \\
\hline $\begin{array}{l}\text { University of Iowa Hospitals and Clinic } \\
\text { Iowa City, Iowa }\end{array}$ & Raymond Tannous, M.D. & CA 29314 \\
\hline $\begin{array}{l}\text { Children's Hospital of Denver } \\
\text { Denver, Colorado }\end{array}$ & David Tubergen, M.D. & CA 28851 \\
\hline
\end{tabular}




\section{References}

1. Cohen ME, Duffner PK, Tebbi CK: Brain Tumors in Children: Diagnosis and Management. In: Cameron K. Tebbi (ed) Major Topics in Pediatric and Adolescent Oncology. G.K. Hall and Co., Boston, 1982

2. McComb RD, Bigner DD: The biology of malignant gliomas - A comprehensive study. Clinical Neuropathology. 3 : 93-106, 1984

3. Rubinstein LJ: Tumors of the Central Nervous System. In: Atlas of tumor pathology, Second series, Fascicle 6. Armed Forces Institute of Pathology, Washington D.C., 1972

4. Sheline GE: Radiation Therapy of Primary Tumors, Seminars in Oncology 64: 29-42, 1975

5. Walker MD: Tumors of the Central Nervous System. In: Arthur S. Levine (ed) Cancer in the Young. Masson Publishing, U.S.A., 1982

6. Chabner BA, Myers CE, Oliverio VT: Clinical pharmacology of anticancer drugs. Seminars in Oncology IV: 165-191, 1977

7. Cole WH: Chemotherapy of Cancer. Lea and Febiger, Philadelphia, 1970

8. Edwards MS, Levin VA, Wilson CB. Brain tumor chemotherapy: An evaluation of agents in current use for phase II and III trials. Cancer Treatment Reports 64: 1179-1205, 1980

9. Shapiro WR. Chemotherapy of primary malignant brain tumors in children. Cancer 35: 965-972, 1975

10. Kerhonan JW, Sayre GP: Tumors of the Central Nervous System. In: Atlas of Tumor Pathology, Section X, Fascicles 35 and 37. Armed Forces Institute of Pathology. Washington D.C., 1952

11. Nelson JS, Tsukada Y, Schoenfeld D, Fulling K, Lamarche $\mathrm{J}$, Peress N: Necrosis as a Prognostic Criterion in Malignant Supratentorial, Astrocytic Gliomas. Cancer 52: 550-554, 1983

12. Fulling KA and Garcia DM. Anaplastic Astrocytoma of the Adult Cerebrum-Prognostic Value of Histologic Features. Cancer 55: 928-931, 1985

13. Pocock SJ, Simon R: Sequential Treatment Assignment with Balancing for Prognostic Factors in the Controlled Clinical Trial. Biometrics 31: 103-115, 1975

14. Byar DP, Simon RM, Friedewald WT, Schlesselman JJ, DeMets DL, Ellenberg JH, Gail MH, Ware JH: Randomized clinical trials: Perspectives on some recent ideas. $\mathrm{N}$ Engl J Med 295: 74-80, 1976

15. Peto R, Pike MC, Armitage P, Breslow NE, Cox DR, Howard SV, Mantel N, McPherson K, Peto J, Smith PG: Design and analysis of randomized clinical trials requiring prolonged observation of each patient. Part II: Analysis and examples. Br J Cancer 35: 1-39, 1977

16. Kalbfleisch JD and Prentice RL: The Statistical Analysis of Failure Time Data. John Wiley and Sons, New York, 1980

17. Peto R, Pike MC, Armitage P, Breslow NE, Cox DR, Howard SV, Mantel N, McPherson K, Peto J, Smith PG: Design and analysis of randomized clinical trials requiring prolonged observation of each patient. Part I: Introduction and design. Br J Cancer 34: 585-612, 1976

18. Simon R, Wittes RE: Methodologic guidelines for reports of clinical trials. Cancer Treatment Reports 69: 1-3, 1985

19. Burger PC, Vogel FS, Green SB, Strike TA: Glioblastoma Multiforme and Anaplastic Astrocytoma. Cancer 56: 11061111,1985

20. Albright LA, Price RA, and Guthkelch AN: Brain stem gliomas of children. Cancer 52: 2313-2319, 1983

21. Afra D, Kocsis B, Dobay J, Eckhardt S: Combined radiotherapy and chemotherapy with dibromudulcitol and $\mathrm{CCNU}$ in the postoperative treatment of malignant gliomas. J Neurosurg 59: 106-110, 1983

22. Chang $\mathrm{CH}$, Horton $\mathrm{H}$, Schoenfeld D, Salazer O, PerezTamayo R, Kramer S, Weinstein A, Nelson JS, Tsukada Y: Comparison of postoperative radiotherapy and combined postoperative radiotherapy and chemotherapy in the multidisciplinary management of malignant gliomas. Cancer 52: 997-1007, 1983

23. EORTC: Effect of CCNU on survival rate of objective remission and duration of free interval in patients with malignant brain glioma - final evaluation. Europ J Cancer 14: 851-856, 1978

24. EORTC: Evaluation of CCNU, VM-26+ CCNU, and procarbazine in supratentorial brain gliomas. J Neurosurg 55: 27-31, 1981

25. Green SB, Byar DP, Walker MD, Pistenmaa DA, Alexander E, Batzdorf U, Brooks WH, Hunt WE, Melaey J, Odom GL, Paoletti P, Ransohoff J, Robertson JT, Selker RG, Shapiro WR, Smith KR, Wilson CB, Strike TA: Comparisons of carmustine, procarbazine, and high-dose methyl prednisolone as additions to surgery and radiotherapy for the treatment of malignant glioma. Cancer Treatment Reports 67: 121-132, 1983

26. Hatlevoll R, Lindegaard K, Hagen S, Kristiansen K, Nesbakken R, Torvik A, Ganz JC, Mella $\mathrm{O}$, Rosengren B, Ringkjob R, Arnasson $\mathrm{O}$, Lindgren S, Lipechi M, Notter G, Littbrand B, Saterborg N, Benediktsson G, Johansson L, Spannare B, Brun A, Berthelsen A, Busch H, Gronbaek E, Rygard J, Haase JP, Lambrethsen E, Midholm S, Sehested P, Heikkinen M, Nystrom S, Taskinen P, Mantyla M, Elgen K, Aaskoven O, DeGaris ST, Jensen RH, Matheson I: Combined modality treatment of operated astrocytomas grade 3 and 4. Cancer 56: 41-47, 1985

27. Reagan TJ, Bisel HG, Childs DS, Layton DD, Rhoton AL, Taylor WF: Controlled study of CCNU and radiation therapy in malignant astrocytoma. J Neurosurg 44: 186-190, 1976

28. Walker MD, Alexander E, Hunt WW, MacCarty CS, Mahaley MS, Mealey J, Norrell HA, Owens G, Ransohoff J, Wilson CB, Gehan EA, Strike TA: Evaluation of BCNU and/or radiotherapy in the treatment of anaplastic gliomas. J Neurosurg 49: 333-343, 1978

29. Walker MD, Green SB, Byar DP, Alexander E, Batzdorf U, Brooks WH, Hunt WE, MacCarty CS, Mahaley MS, Mealey J, Owens G, Ransohoff J, Robertson JT, Shapiro 
WR, Smith KR, Wilcon CB, Strike TA: Randomized comparisons of radiotherapy and nitrosoureas for the treatment of malignant glioma after surgery. N Engl J Med 303: 13231329,1980

30. Stenning SP, Freedman LS, Bleehen NM: An overview of published results from randomized studies of nitrosoureas in primary high grade malignant glioma. Br J Cancer 56: $89-90,1987$

Address for offprints: Childrens Cancer Study Group, 199 N. Lake Ave., Third Floor, Pasadena, CA, 91101, USA 